\title{
BMJ Open Real-world outcomes comparison among adults with atrial fibrillation undergoing catheter ablation with a contact force porous tip catheter versus a second-generation cryoballoon catheter: a retrospective analysis of multihospital US database
}

\author{
Alexandru Costea, ${ }^{1}$ Laura Goldstein, ${ }^{2}$ Sonia Maccioni, ${ }^{2}$ Iftekhar Kalsekar, ${ }^{3}$ \\ Rahul Khanna (10 ${ }^{3}$
}

To cite: Costea A, Goldstein L, Maccioni S, et al. Real-world outcomes comparison among adults with atrial fibrillation undergoing catheter ablation with a contact force porous tip catheter versus a secondgeneration cryoballoon catheter: a retrospective analysis of multihospital US database. BMJ Open 2020;10:e035499. doi:10.1136/ bmjopen-2019-035499

- Prepublication history and additional material for this paper are available online. To view these files, please visit the journal online (http://dx.doi. org/10.1136/bmjopen-2019035499).

Received 04 November 2019 Revised 12 May 2020 Accepted 14 July 2020

Check for updates

(C) Author(s) (or their employer(s)) 2020. Re-use permitted under CC BY-NC. No commercial re-use. See rights and permissions. Published by BMJ.

For numbered affiliations see end of article.

Correspondence to

Dr Rahul Khanna;

rkhann14@its.jnj.com

\section{ABSTRACT}

Objective To compare real-world clinical and economic outcomes among atrial fibrillation (AF) patients undergoing cardiac ablation with the contact forcesensing porous tip THERMOCOOL SMARTTOUCH SF (STSF) catheter versus the Arctic Front Advance Cryoballoon (AFA-CB) catheter.

Design Retrospective, observational cohort study. Setting Premier Healthcare Database (PHD), between 1 September 2016 and 30 June 2018.

Participants Patients with AF ( $\geq 18$ years) were included if they had an index ablation procedure performed using the STSF catheter or AFA-CB catheter at a US hospital that consistently provided inpatient and outpatient data to PHD in the 12-month preindex period. Using 1:1 propensity score matching, patient groups were matched on study covariates.

Primary and secondary outcome measures Cost, length of stay (LOS), readmissions, direct current cardioversion (DCCV) and reablation outcomes were compared between matched cohorts of STSF and AFA-CB patients.

Results A total of 3015 patients with AF met the study criteria, of which 1720 had ablation using the STSF catheter and 1295 had ablation using the AFA-CB catheter. In the propensity-matched sample, patients receiving ablation with the STSF catheter had $\sim 17 \%$ lower total costs (US\$23 096 vs US\$27 682, $p \leq 0.0001$ ) and 27\% lower supply costs (US\$10 208 vs US $\$ 13816, p \leq 0.0001$ ) versus patients receiving ablation with the AFA-CB catheter. A significantly lower likelihood of 4-month to 6-month cardiovascular-related readmission (OR 0.460, $95 \% \mathrm{Cl} 0.220$ to 0.959 ) was associated with the STSF catheter versus the AFA-CB catheter. No significant differences in LOS, room and board cost, 4-month to 6-month all-cause and AF-related readmissions, DCCV and reablation were observed among technologies. Sensitivity analysis restricting patient sample by provider ablation volume demonstrated similar results.
Strengths and limitations of this study

The Premier Healthcare Database represents a large (>700 US acute care hospitals) and diverse data source.

- The use of propensity score matching methodology alleviated selection bias.

- Generalised estimating equation allowed for adjustment of hospital clustering.

- Unmeasured confounders could have influenced study results.

- Factors including atrial fibrillation severity, drug use and procedural elements could not be assessed and controlled.

Conclusion Lower index ablation total and supply costs were observed among patients with AF undergoing cardiac catheter ablation using the STSF catheter versus the AFACB catheter.

\section{INTRODUCTION}

Atrial fibrillation (AF) is the most common cardiac arrhythmia and a major risk factor for stroke, ${ }^{1}$ necessitating timely management and treatment. An effective treatment option for patients with AF who are refractory or intolerant to pharmacotherapy is catheter ablation. $^{2-4}$ Ablation treatment with the use of radiofrequency ( $\mathrm{RF}$ ) catheters is one of the most effective options for patients with paroxysmal AF and involves electrical isolation of the pulmonary veins (PVs). Over the past few years, significant improvements in RF technology have been made with the advent of advanced porous tip and contact force (CF)-sensing technology. RF catheters 
with advanced porous tip technology allow for uniform cooling of cardiac tissue at the site of the ablation and lower fluid delivery, while catheters with CF technology significantly improve the ability to create durable lesions by providing information on real-time catheter-to-tissue interaction. A reduction in overheating risk associated with uniform cooling of advance porous tip technology could potentially allow for creation of large lesions with greater depth by allowing delivery of greater quantities of RF power. Further, the reduced fluid delivery associated with advance porous tip technology could offer benefit to patients with heart failure and ventricular dysfunction as these patients are particularly susceptible to fluid overload. ${ }^{5}$

The THERMOCOOL SMARTTOUCH SF (STSF) catheter (Biosense Webster, Irvine, California, USA) with CF-sensing and advanced 56-hole porous tip irrigation allows for durable lesion creation, as well as uniform cooling and reduced fluid delivery. A recent study compared safety and clinical outcomes among symptomatic, drug-refractory, paroxysmal or early persistent patients with AF who underwent catheter ablation using the STSF catheter $(n=75)$ versus a historical cohort of patients who had ablation with the THERMOCOOL SMARTTOUCH (ST) catheter $(\mathrm{n}=35)$, a CF catheter with conventional tip irrigation. ${ }^{5}$ A $51 \%$ reduction in fluid delivery was achieved with the STSF catheter versus the ST catheter $(\mathrm{p}<0.01)$. The 12-month arrhythmia-free survival rates were $79.9 \%$ (95\% CI $70.4 \%$ to $90.4 \%$ ) for the STSF cohort versus $66.7 \%$ (95\% CI $50.2 \%$ to $88.5 \%$ ) for the ST cohort $(\mathrm{p}=0.18)$.

Catheter ablation can also be performed using a cryoballoon (CB) catheter. As with RF catheters, technological improvements have been associated with $\mathrm{CB}$ catheters. The second-generation $\mathrm{CB}$ catheter (Arctic Front Advance $\mathrm{CB}$ catheter (AFA-CB), Medtronic, Minneapolis, Minnesota, USA) provides a more homogeneous and larger zone of cooling compared with the first-generation Arctic Front catheter (AF-CB). Studies have reported improved outcomes among paroxysmal AF patients, including greater 12-month freedom from $\mathrm{AF}$ and lower procedure and fluoroscopy times with second-generation AFA-CB catheter compared with the first-generation AF-CB catheter. $^{67}$

A few studies have compared the use of the RF catheters with the AF-CB/AFA-CB catheters. ${ }^{8-12}$ In the Fire and Ice Trial, which was a multicentre randomised controlled trial comparing RF ablation (using first generation THERMOCOOL, THERMOCOOL SF, or ST catheters) with CB ablation (AF-CB or AFA-CB), no significant difference in safety and efficacy was found between the two groups of catheters in the ablation of drug-refractory paroxysmal AF. ${ }^{10} 11$ The primary efficacy endpoint (a composite endpoint of AF recurrence, atrial flutter or atrial tachycardia occurrence, antiarrhythmic drug prescription or repeat ablation) did not differ between $\mathrm{RF}$ and $\mathrm{CB}$ ablation arms $(35.9 \%$ vs $34.6 \%$, respectively, HR $0.96,95 \%$ CI 0.76 to 1.22$)$. In their prospective single-centre study comparing the AFA-CB catheter with the ST catheter, Jourda and colleagues ${ }^{8}$ found no significant difference in the 12-month AF recurrence among the two groups (HR $1.20,95 \%$ CI 0.50 to 2.90 ). In one study using a nationally representative hospital database, healthcare resource utilisation was compared among patients with AF undergoing ablation using the $\mathrm{ST}$ catheter versus $\mathrm{AF} / \mathrm{AFA}-\mathrm{CB}$ catheter. ${ }^{9}$ The ST catheter $(\mathrm{n}=1409)$ was associated with significantly lower total $(\sim 7 \%)$ and supply $(\sim 13 \%)$ costs and a significantly lower likelihood of 4-month to 12-month all-cause and cardiovascular (CV)-related readmission compared with the AF/AFA-CB catheter $(\mathrm{n}=2306)$. In addition to clinical and economic outcomes, studies have also compared efficiency endpoints, such as procedure and fluoroscopy times between the two technologies. In one such prior study, the authors compared procedure and fluoroscopy time between the two technologies in a single-centre study, which showed longer procedure time for $\mathrm{CB}$ ablation compared with RF ablation ( 171 vs $126 \mathrm{~min}, \mathrm{p}<0.0001$ ), with no significant difference in fluoroscopy time (32 vs $29 \mathrm{~min}, \mathrm{p}=0.39) .{ }^{12}$ The authors alluded that the longer procedure time for $\mathrm{CB}$ ablation could be attributed to the time requirements for freeze-thaw-freeze applications, higher use of protamine and the need for additional RF point-by-point use. The need for additional RF point-by-point catheter ablation with $\mathrm{CB}$ catheter not only adds to procedure time but also adds to the cost of the overall procedure for hospitals. Other studies have reported shorter ablation and procedure times and longer fluoroscopy time associated with the use of second-generation CB catheter compared with RF catheter. ${ }^{13}$

To our knowledge, no study to date has compared outcomes for AF ablation among the $\mathrm{CF}$ porous tip STSF catheter versus the AFA-CB catheter in a real-world setting. The number of individuals with AF in the USA is expected to increase to $6-12$ millions by the year $2050 .^{14}$ The rising AF prevalence coupled with increasing scarcity of healthcare resources requires a thorough assessment of the health outcomes and cost differences among AF ablation technologies. The objective of this retrospective, observational study was to compare cost, length of stay (LOS), readmissions, direct current cardioversion (DCCV) and repeat ablation among patients with AF who underwent ablation using the STSF catheter versus the AFA-CB catheter in a real-world setting.

\section{METHODS}

\section{Data source}

This was an observational cohort study using data from the Premier Healthcare Database (PHD). The PHD contains complete clinical coding, hospital cost and patient billing data from a nationally representative sample (as per bed size, geographic region, urban/rural location and teaching status) of more than 700 acute care hospitals and represents roughly $20 \%$ of all inpatient discharges in the USA. 


\section{Study sample}

Patients who had an elective ablation procedure between 1 September 2016 and 30 June 2018 were identified by either the -Current Procedural Terminology (CPT) code 93656 with primary or secondary AF diagnosis, or equivalent ICD-10 Procedure code for an ablation procedure with a primary diagnosis of AF. The first observed ablation (inpatient or outpatient) meeting these criteria was designated as the index hospital ablation. Eligible patients were required to be at least 18 years of age at the time of the index procedure. Patients who had an index ablation procedure performed at a hospital that consistently provided inpatient and outpatient data to PHD in the 12-month preindex period were identified. Among these patients, those who had surgical ablation, valvular procedures or a left atrial appendage occlusion procedure in the 12-month preindex period were excluded. Patients who had a total cost or supply cost listed as US $\$ 0$ or those who had the room and board cost listed as US $\$ 0$ (for inpatient ablations) were also excluded. Patients with AF who underwent catheter ablation using either the porous tip CF STSF catheter or the AFA-CB catheter (with or without additional point-by-point catheter use) were identified using text search strategy of hospital charge descriptors. We used combination of device name and catalogue numbers to identify the use of study devices in PHD. The two groups of patients were then matched 1:1 on study covariates using propensity matching.

\section{Study covariates}

Patient demographics including age (18-49, 50-59, $60-69, \geq 70$ years), gender (male, female), race (white, non-white) and marital status (married, single, other) and payer type (commercial, Medicare, Medicaid, others) were collected at index hospitalisation.

The Extended-Charlson Comorbidity Index (CCI) score was used for comorbidity assessment. ${ }^{15} 16$ In addition, patients' $\mathrm{CHA}_{2} \mathrm{DS}_{2}$ VASc score, presence of individual comorbidities including obstructive sleep apnoea, obesity, diabetes, hypertension, chronic pulmonary disease, kidney disease/renal disease, congestive heart failure (CHF), atrial flutter, other arrhythmias, valvular disease, cardiomyopathy and ischaemic heart disease (with or without myocardial infarction (MI)) were assessed.

Hospital admission setting (inpatient or outpatient) and year of index ablation were also evaluated. Hospital characteristics including teaching status, hospital bed size (under 300 beds, 300-499 beds and $\geq 500$ beds), hospital region (midwest, northeast, south, west) and the specialty of the physician performing the procedure were also identified. Volume of total hospital ablation procedures (converted to categorical variable using median split) performed in the 12-month preindex ablation period was also assessed.

\section{Study outcomes}

Hospital costs (total, supply, and room and board (for inpatient ablations)), inpatient LOS, readmissions (all-cause, CV-related and AF-related), repeat ablation and DCCV were examined and compared among the two catheter groups. Outcomes including total and supply costs were examined for the final sample of propensitymatched patients. The total cost refers to sum of all billable items including supply cost, labour cost, laboratory cost, pharmacy cost, and room and board cost during the procedure LOS and are reported by the hospital. LOS and room and board cost were assessed for patients who had inpatient ablation. All-cause, CV-related and AF-related readmissions, repeat ablation and DCCV were measured during the postblanking 4-month to 6-month period after discharge from the index ablation procedure, with the first 3 months being considered as blanking period. Guidelines recommend that a blanking period of 3 months be considered when examining efficacy outcomes for cardiac ablation, with recurrence during the first 3 months not to be considered as failure. ${ }^{4}$ At the time of study initiation, we had data available until mid-2018, and given the release of STSF catheter in second half of 2016, we had to restrict the follow-up period to 6 months, with first 3 months as blanking period. Costs were adjusted for inflation and are reported in 2017 US dollars.

\section{Patient and public involvement}

Patients and public were not directly involved at any stage in the research process.

\section{Statistical analysis}

A 1:1 propensity score matching, using the greedy nearest neighbour matching without replacement within 0.10 calliper width, was performed to match patients in the STSF and AFA-CB catheter group on study covariates including patient demographic and comorbid characteristics, procedural characteristics and provider characteristics. Postmatch balance of covariates was examined using standardised mean differences (SMDs), in addition to $\mathrm{p}$ values from bivariate comparison of study covariates among the two study cohorts (prematch and postmatch). It should though be noted that SMDs are not influenced by sample size unlike traditional (t-test, $\chi^{2}$ ) statistical techniques. ${ }^{17}$ Four sets of propensity models were run to examine cost (total and supply), LOS and room and board cost, readmissions (all-cause, CV-related, AF-related), and DCCV and reablation, respectively. For total and supply cost, which were assessed during the index ablation, propensity matching was run on the final prematch sample of STSF and AFA-CB patients undergoing ablation in an inpatient or outpatient setting. For LOS and room and board cost, assessed during index ablation, propensity matching was run on the prematch sample of STSF and AFA-CB patients who had ablation in an inpatient setting. Inpatient readmissions (all-cause, CV-related, AF-related), DCCV and reablation were examined in patients who underwent index ablation in hospitals that continuously provided inpatient and outpatient data to PHD. 
A generalised estimating equation (GEE) model with an exchangeable correlation structure with log link and gamma distribution function was used for cost comparison in the matched cohorts. For LOS comparisons, GEE with log link and negative binomial distribution was used. To examine readmissions (all-cause, CV-related, AF-related), DCCV and reablation, GEE with logit link and binomial distribution was used. Potential hospital-level clustering was adjusted for in GEE analyses. Further, any covariates that emerged significantly different postmatching, that is, having SMD $\geq 0.10$ or $\leq-0.10$, were also controlled for in GEE analyses.

\section{Sensitivity analysis}

Studies have reported that the volume of AF ablation procedures performed by a hospital is associated with patient outcomes, with centres performing 100 procedures or more reported to have better outcomes. ${ }^{18}$ To control for the effect of volume, we performed a sensitivity analysis by restricting the sample across the STSF and AFA-CB catheter group to those patients who had their index ablation procedure performed at hospitals that had at least 100 or more total ablations in the preindex 12-month period. As with the main analysis, propensity matching was performed, and study outcomes were then compared in the matched cohort.

\section{RESULTS}

\section{Patient attrition}

Table 1 depicts the patient attrition based on study inclusion and exclusion criteria. Among the 3015 patients that met the study criteria, 1720 had AF ablation using the STSF catheter and 1295 had AF ablation using the AFA-CB catheter (with or without additional point-bypoint catheter use).

\section{Study characteristics}

Online supplementary table 1 describes patient demographic and comorbidity characteristics and hospital characteristics. Among both STSF and AFA-CB catheter patients, a majority were greater than 60 years of age, male, white and married. A higher proportion of STSF patients had a CCI score of $\geq 2(27.21 \%$ vs $20.00 \%$, SMD $=0.17)$ compared with AFA-CB patients. In addition, a higher proportion of patients in the STSF catheter group versus the AFA-CB catheter group had sleep apnoea $(30.47 \%$ vs $19.38 \%, \mathrm{SMD}=0.25)$, obesity $(25.64 \%$ vs $18.61 \%$, $\mathrm{SMD}=0.17)$, atrial flutter $(43.72 \%$ vs $27.88 \%, \mathrm{SMD}=0.33)$, other arrhythmias $(19.42 \%$ vs $15.52 \%, \quad \mathrm{SMD}=0.10)$, valvular disease $(22.09 \%$ vs $12.51 \%, \mathrm{SMD}=0.25)$, cardiomyopathy $(14.36 \%$ vs $9.65 \%, \mathrm{SMD}=0.14)$, ischaemic heart disease/MI (25.47\% vs $20.08 \%$, SMD $=0.12)$, hypertension $(69.53 \%$ vs $59.46 \%, \mathrm{SMD}=0.21)$ and $\mathrm{CHF}(23.49 \%$ vs $19.31 \%, \mathrm{SMD}=0.10)$. The prematch and postmatch characteristics and SMDs among the STSF and AFA-CB catheter for the total cost and supply cost; LOS and room and board cost; and readmission, DCCV and reablation

Table 1 Patient attrition in the overall study sample

\begin{tabular}{|c|c|c|}
\hline Step & Criteria & $\begin{array}{l}\text { Eligible patients } \\
\text { (n) }\end{array}$ \\
\hline 1 & $\begin{array}{l}\text { Patients with an ablation procedure listed with a primary diagnosis (with ICD-10 ablation } \\
\text { procedure codes)/primary or secondary diagnosis (with CPT 93656) of AF during } 1 \text { September } \\
2016-30 \text { June } 2018\end{array}$ & 18662 \\
\hline 2 & Patients $\geq 18$ years during index admission & 18658 \\
\hline 3 & Patients with an elective ablation procedure & 14196 \\
\hline 4 & $\begin{array}{l}\text { Patients with the index procedure at hospitals that provided data (inpatient and outpatient) for } \\
\text { at least } 12 \text { months during the preindex period in the Premier database }\end{array}$ & 12826 \\
\hline 5 & $\begin{array}{l}\text { Exclusion of patients with a procedural code for catheter ablation performed in the 12-month } \\
\text { preindex period }\end{array}$ & 12377 \\
\hline 6 & $\begin{array}{l}\text { Exclusion of patients with a procedural code for surgical ablation during the 12-month } \\
\text { preindex or index admission }\end{array}$ & 12096 \\
\hline 7 & $\begin{array}{l}\text { Exclusion of patients with a procedural code for valvular procedures or atrioventricular node } \\
\text { ablation during the } 12 \text {-month preindex period }\end{array}$ & 11849 \\
\hline 8 & $\begin{array}{l}\text { Exclusion of patients with a procedural code for left atrial appendage occlusion during the } \\
12 \text {-month preindex period }\end{array}$ & 11726 \\
\hline 9 & $\begin{array}{l}\text { Patients who had total cost and supply cost }>0 \text {, and for patients who had ablation in an } \\
\text { inpatient setting, the room and board cost }>0\end{array}$ & 11630 \\
\hline 10 & $\begin{array}{l}\text { Patients with either a STSF catheter (THERMOCOOL SMARTTOUCH SF) or CB catheter } \\
\text { (Arctic Front Advance) device record }\end{array}$ & $\begin{array}{l}\text { STSF }=1720 \\
\text { CB }=1295\end{array}$ \\
\hline
\end{tabular}

AF, atrial fibrillation; CB catheter, Artic Front Advance catheter; STSF catheter, THERMOCOOL SMARTTOUCH SF catheter; CPT, Current Procedural Terminology ; ICD, International Classification of Diseases. 
comparisons are described in online supplementary tables 1-3, respectively. The SMDs for covariates among the matched sample of STSF and AFA-CB patients $(n=848$ in each cohort) for the total cost and supply cost comparison were within the 0.10 to -0.10 range, indicating a wellmatched cohort (online supplementary table 1). When examining the SMDs for study covariates in the matched sample for the LOS and room and board cost comparison, several covariates fell outside the 0.10 to -0.10 range (online supplementary table 2). Considering the limited sample size in the match cohort ( $\mathrm{n}=92$ in each cohort), we did not adjust for these significant variables in the GEE analysis for LOS and room and board cost comparison. In the matched sample of STSF and AFA-CB patients $(n=568$ in each cohort; online supplementary table 3$)$ for inpatient readmission, DCCV and reablation outcome comparison, SMDs were within the 0.10 to -0.10 range for all study covariates except ischaemic heart disease comorbidity ( $\mathrm{SMD}=0.1091)$, bed size $0-299(\mathrm{SMD}=0.1045)$ and teaching status ( $\mathrm{SMD}=0.1558)$. We adjusted for the three covariates (ischaemic heart disease, bed size and teaching status) in the GEE model when comparing readmissions, DCCV and reablation among the matched STSF and AFA-CB patients.

Online supplementary table 4-6 depict the prematch and postmatch characteristics and standardised differences for total and supply cost, LOS and room and board cost, and readmission, DCCV, and reablation comparisons, respectively, for the sensitivity analysis sample. Similar to the primary analysis, a comparison of study covariates from the sensitivity analysis sample showed that the STSF catheter group had a higher comorbidity burden in comparison with the AFA-CB catheter group.

\section{Study outcomes}

Hospital costs

After propensity matching for index cost (total and supply) comparison, 848 patients were identified in each of the STSF and AFA-CB catheter group. GEE analysis results showed that the STSF cohort had $\sim 17 \%$ lower total costs ( $\$ 23096$ vs $\$ 27682, \mathrm{p} \leq 0.0001)$ and $\sim 27 \%$ lower supply costs (US\$10 208 vs US $\$ 13816$, p $\leq 0.0001$ ) compared with the AFA-CB catheter cohort (table 2A). In the postmatch sensitivity analysis sample $(\mathrm{n}=75$ in each cohort) including the STSF and AFA-CB cohort from high-volume centres, the STSF catheter was associated with $\sim 15 \%$ lower total costs (US $\$ 22242$ vs US $\$ 26$ $003, \mathrm{p} \leq 0.0001$ ) and $\sim 24 \%$ lower supply costs (US\$9951 vs US $\$ 12935, \mathrm{p} \leq 0.0001)$ versus the AFA-CB catheter (table 2B).

\section{LOS and room and board cost}

LOS (2.98 (95\% CI 2.41 to 3.68 ) vs 2.41 days (95\% CI 1.98 to 2.94$), \mathrm{p}=0.1465)$ and room and board costs (US\$3404 vs US $\$ 3391, \mathrm{p}=0.9845$ ) did not differ significantly between STSF and AFA-CB catheter patients $(n=92$

Table 2 Regression adjusted total index ablation costs and supply costs for inpatient and outpatient ablations and LOS (days) and room and board costs for inpatient ablations only in patients with AF undergoing ablation using the STSF catheter compared with the AFA-CB catheter in the (A) primary analysis and (B) sensitivity analysis (preindex ablation volume $\geq 100$ )

\section{(A) Primary analysis}

\begin{tabular}{|c|c|c|c|}
\hline Total index ablation and supply cost ${ }^{\star}$ & $\begin{array}{l}\text { STSF catheter } \\
n=848\end{array}$ & $\begin{array}{l}\text { CB catheter } \\
n=848\end{array}$ & Exponentiated ratio $(\mathrm{Cl})$ \\
\hline Total index ablation cost & US\$23096 & US\$27 682 & 0.83 (0.78 to 0.88$)$ \\
\hline LOS and room and board cost (inpatient only) ${ }^{*} \dagger$ & $\begin{array}{l}\text { STSF catheter } \\
n=92\end{array}$ & $\begin{array}{l}\text { CB catheter } \\
n=92\end{array}$ & Exponentiated ratio $(\mathrm{Cl})$ \\
\hline LOS (days) & 2.98 & 2.41 & 1.23 (0.92 to 1.63$)$ \\
\hline
\end{tabular}

(B) Sensitivity analysis (preindex ablation volume $\geq 100$ )

\begin{tabular}{|c|c|c|c|}
\hline Total index ablation and supply cost* & $\begin{array}{l}\text { STSF catheter } \\
n=754\end{array}$ & $\begin{array}{l}\text { CB catheter } \\
n=754\end{array}$ & Exponentiated ratio $(\mathrm{Cl})$ \\
\hline Total index ablation cost & US\$22 242 & US\$26 003 & 0.85 (0.81 to 0.90$)$ \\
\hline LOS and room and board cost (inpatient only) ${ }^{\star} \dagger$ & $\begin{array}{l}\text { STSF catheter } \\
n=73\end{array}$ & $\begin{array}{l}\text { CB catheter } \\
n=73\end{array}$ & Exponentiated ratio $(\mathrm{Cl})$ \\
\hline LOS (days) & 2.96 & 2.46 & $1.20(0.80$ to 1.79$)$ \\
\hline
\end{tabular}

*Propensity score-matched analysis; results based on GEE model with log link and gamma distribution.

†Several variables were significantly different between the STSF catheter groups versus the CB catheter group for the inpatient ablation sample; however, considering the low sample size postmatching, GEE model was run without adjusting for these differences. AF, atrial fibrillation; CB catheter, Artic Front Advance catheter; GEE, generalised estimating equation; LOS, length of stay; ; STSF catheter, THERMOCOOL SMARTTOUCH SF catheter. 
Table 3 Likelihood of inpatient readmissions, DCCV and reablation during the 4-month to 6-month postindex period in patients with AF undergoing ablation using STSF catheter or AFA-CB catheter in the bivariate analysis and regression analyses in the primary analysis

\begin{tabular}{lllll} 
Inpatient readmission & $\begin{array}{l}\text { STSF catheter } \\
\mathbf{n = 5 6 8}\end{array}$ & $\begin{array}{l}\mathbf{C B} \text { catheter } \\
\mathbf{n = 5 6 8}\end{array}$ & P value & $\begin{array}{l}\text { OR (95\% Cl)* } \\
\text { STSF catheter (n=568) vs } \\
\text { CB catheter (n=568) }\end{array}$ \\
\hline All-cause readmission & $3.35 \%$ & $4.58 \%$ & 0.2870 & $0.69(0.38$ to 1.24$)$ \\
CV-related readmission & $1.76 \%$ & $3.35 \%$ & 0.0905 & $0.46(0.22$ to 0.95$)$ \\
AF-related readmission & $1.06 \%$ & $2.29 \%$ & 0.1053 & $0.41(0.16$ to 1.05$)$ \\
DCCV and reablation & & & & \\
DCCV & $2.99 \%$ & $2.82 \%$ & 0.8598 & $1.04(0.60$ to 1.80$)$ \\
Reablation & $1.58 \%$ & $1.58 \%$ & 1.0000 & $0.98(0.47$ to 2.07$)$ \\
\hline
\end{tabular}

${ }^{*}$ Results based on generalised estimating equation with logit link and binomial distribution; model adjusted for teaching status, bed size and ischaemic heart disease with or without myocardial infarction, which emerged as significantly different between the STSF catheter group versus the $C B$ catheter group in the matched sample.

AF, atrial fibrillation; CB catheter, Artic Front Advance catheter; STSF catheter, THERMOCOOL SMARTTOUCH SF catheter; CV, cardiovascular; DCCV, direct current cardioversion.

in each cohort) (table 2A). Further, no significant difference in LOS (2.96 (95\% CI 2.26 to 3.87$)$ vs 2.46 (95\% CI 1.88 to 3.24 ) days, $\mathrm{p}=0.3729$ ) and room and board costs (US $\$ 3520$ vs US $\$ 3412, \mathrm{p}=0.8760$ ) was observed for the STSF and AFA-CB patients ( $\mathrm{n}=73$ in each cohort) in the sensitivity analysis (table 2B).

\section{Inpatient readmissions}

There were 568 patients in each of the STSF and AFA-CB catheter groups in the propensity-matched sample for inpatient readmission comparison. Results from GEE analysis showed $54 \%$ lower likelihood (OR 0.46, 95\% CI 0.22 to 0.95 ) of CV-related inpatient admissions in the 4-month to 6-month postindex ablation period among STSF patients compared with AFA-CB patients. No significant difference in all-cause inpatient readmission (OR $0.69,95 \%$ CI 0.38 to 1.24$)$ and AF-related inpatient readmission (OR $0.41,95 \%$ CI 0.16 to 1.05 ) was observed among patients in the STSF and AFA-CB catheter groups, as the $95 \%$ CIs for the difference were wide (table 3 ).

In the propensity-matched sample for sensitivity analysis, there were 465 patients in each of the STSF and AFA-CB catheter groups. In the 4-month to 6-month postindex ablation period, STSF patients were found to have $66 \%$ lower likelihood (OR $0.34,95 \%$ CI 0.17 to 0.69) of CV-related readmissions in comparison with the AFA-CB patients. All-cause readmissions (OR 0.63, 95\% CI 0.38 to 1.05) and AF-related readmissions (OR 0.63, $95 \%$ CI 0.26 to 1.48 ) did not differ significantly among the two groups of patients (table 4).

\section{DCCV and reablation}

There were 568 propensity-matched patients each in the primary analysis comparing DCCV and reablation among the STSF and AFA-CB catheter group. No significant differences in DCGV (OR 1.043, 95\% CI 0.602 to 1.807)

Table 4 Likelihood of inpatient readmissions, DCCV and reablation during the 4-month to 6-month postindex period in patients with AF undergoing ablation using STSF catheter or AFA-CB catheter in the bivariate analysis and regression analyses in the sensitivity analysis (preindex ablation volume $\geq 100$ )

\begin{tabular}{|c|c|c|c|c|}
\hline Inpatient readmission & $\begin{array}{l}\text { STSF catheter } \\
\mathrm{n}=465\end{array}$ & $\begin{array}{l}\text { CB catheter } \\
n=465\end{array}$ & $P$ value & $\begin{array}{l}\text { OR }(95 \% \text { Cl })^{*} \\
\text { STSF catheter }(n=465) \text { vs } \\
\text { CB catheter }(n=465)\end{array}$ \\
\hline All-cause readmission & $2.80 \%$ & $4.52 \%$ & 0.1622 & 0.63 (0.38 to 1.05$)$ \\
\hline CV-related readmission & $1.51 \%$ & $3.01 \%$ & 0.1223 & 0.34 (0.17 to 0.69$)$ \\
\hline AF-related readmission & $1.29 \%$ & $1.72 \%$ & 0.5902 & 0.63 (0.26 to 1.48$)$ \\
\hline \multicolumn{5}{|l|}{ DCCV and reablation } \\
\hline DCCV & $3.23 \%$ & $2.58 \%$ & 0.5579 & 1.29 (0.63 to 2.62$)$ \\
\hline Reablation & $1.51 \%$ & $1.72 \%$ & 0.7946 & 0.98 (0.46 to 2.10$)$ \\
\hline
\end{tabular}

${ }^{*}$ Results based on generalised estimating equation with logit link and binomial distribution; model adjusted for provider region and setting, which emerged as significantly different between the STSF catheter group versus the CB catheter group in the matched sample. AF, atrial fibrillation; CB catheter, Artic Front Advance catheter; CV, cardiovascular; DCCV, direct current cardioversion; STSF catheter, THERMOCOOL SMARTTOUCH SF catheter. 
and reablation (OR 0.98 , 95\% CI 0.47 to 2.07 ) outcomes were observed among the STSF and AFA-CB patients (table 3).

In sensitivity analysis, 465 patients were identified in each of the STSF and AFA-CB catheter groups. Results from GEE analysis did not reveal any significant difference in the likelihood of DCCV (OR 1.29, 95\% CI 0.63 to 2.62) or reablation (OR $0.98,95 \%$ CI 0.46 to 2.10 ) among STSF and AFA-CB patients (table 4).

\section{DISCUSSION}

This real-world analysis of a nationally representative multihospital database examined the differences in cost, readmissions, DCCV and reablation among patients undergoing AF ablation using a CF porous tip STSF catheter compared with the AFA-CB catheter. Patients who had undergone RF ablation using the STSF catheter had significantly lower total costs and supply costs, and lower likelihood of CV-related inpatient readmissions in comparison with patients who had AF ablation using the AFA-CB catheter.

When observing the differences in overall comorbidity burden (CCI) score and prevalence of comorbidities, patients who underwent AF ablation using the STSF catheter were found to be sicker than those who had ablation using the AFA-CB catheter. This underlying pattern was also recently reported in the study comparing ST catheter use versus $\mathrm{AF} / \mathrm{AFA}-\mathrm{CB}$ catheter use, ${ }^{9}$ suggesting a preference for an advanced RF ablation catheter (ie, ST or STSF catheter) over a CB catheter in patients with higher comorbidity burden. The higher comorbidity burden among the STSF catheter group likely reflects an underlying channelling of higher risk patients to the STSF catheter versus AFA-CB catheter. Another reason for this underlying channelling of higher risk patients may be that the STSF focal catheter provides improved flexibility to ablate additional areas outside of the PV for patients with other arrhythmias (including atrial flutter) in addition to $\mathrm{AF}$ or for patients with $\mathrm{AF}$ with non-PV triggers. Irrespective of the underlying reason, this differential in patient characteristics further necessitates the need for selection bias adjustment in any comparison involving the two technologies.

Although a few studies have compared the RF catheters (including RF CF catheter) and CB catheters and have reported similar effectiveness for the two technologies, ${ }^{8-12} 19-22$ this is one of the first studies comparing the porous tip CF STSF catheter with AFA-CB catheter. Further, current published studies comparing the two technologies have suffered from limited generalisability, as they have been restricted to very few centres and may not be fully representative of the difference in the impact of the two technologies among patients with $\mathrm{AF}$ in a realworld setting. Most recently, lower total costs and supply costs, and a significantly lower likelihood of 4-month to 12-month all-cause and CV-related inpatient readmissions were demonstrated with the ST catheter compared with
AF/AFA-CB catheter in a real-world study using a multihospital US database. ${ }^{9}$ Our results build on this earlier study (of ST vs AF/AFA-CB comparison), by including a CF catheter with an advanced irrigation system (STSF) and comparing it with the AFA-CB catheter. As with the earlier study, we observed significant cost savings and a lower likelihood of CV-related readmission associated with the RF STSF catheter compared with the CB catheter. Study results showed an approximate US\$4586 (95\% CI US $\$ 3973$ to US\$5277) in hospital cost savings associated with the use of the STSF catheter compared with the AFA-CB catheter (with or without additional point-bypoint catheter use). These cost savings were largely attributable to the use of additional point-by-point catheter use alongside AFA-CB catheter. Almost 55\% of the AFA-CB sample had additional point-by-point catheter use. To better understand the cost differential between the two catheters and influence of additional point-by-point catheter use with AFA-CB catheter, we compared index admission cost between STSF patients and AFA-CB patients who did not have additional point-by-point catheter use $(\mathrm{n}=581$; results available on request $)$. As with main study analysis, when comparing cost among a propensity-matched sample ( $\mathrm{n}=432$ in each cohort) of patients who had ablation using either the STSF or AFA-CB catheter (without additional point-by-point catheter use), STSF use was still associated with significantly lower cost compared with the AFA-CB (US\$23 105 vs US\$25 412; p=0.0082). Although the US\$2300 cost savings observed when comparing the STSF catheter with AFA-CB catheter without any additional point-by-point catheter use is lower than the roughly US $\$ 4600$ savings observed in the main analysis comparison, it still highlights the economic savings generated by the use of the STSF catheter compared with AFA-CB catheter. These results demonstrate that newer RF catheter ablation technology continues to provide economic savings, as with earlier generations of RF catheters when compared with the $\mathrm{CB}$ catheter. As healthcare dollars become scarce, providers could generate significant cost savings by using advanced RF ablation catheters such as STSF compared with the CB catheter.

Patients who had AF ablation using the advanced irrigated STSF catheter had 54\% lower odds of CV-related readmission compared with the AFA-CB catheter group in the postblanking 4-month to 6-month period. There were no significant differences in the likelihood of allcause and AF-related readmissions and DCCV and reablation between the STSF catheter and the AFA-CB catheter groups. Results from sensitivity analyses, wherein patient sample was restricted to high-volume centres, were similar. The AF-related readmissions seem to be the major driver of CV-related readmissions in both set of patients. Although the likelihood of AF-related readmission did not emerge to be statistically significantly different among the STSF and AFA-CB patients in this study, it should be noted that the rate of readmission was almost half in the former group compared with the latter $1.06 \%$ vs $2.29 \% ; \mathrm{p}=0.1053)$. In an earlier study comparing the 
ST catheter with AF / AFA-CB catheter, ${ }^{9}$ significantly lower odds of CV-related readmissions were observed in the postblanking 4-month to 12-month period. These results indicate that advanced RF technology potentially offers significant reductions in disease burden compared with CB technology. Despite the potential clinical improvements associated with STSF catheter compared with AFA-CB catheter, it should be noted that the overall rate of AF-related and CV-related readmission was low, and no significant difference was observed among the two technologies in rate of readmission. Further research is needed to understand true differences in AF recurrence, including recurrence identified by ECG/Holter monitor, between the STSF and AFA-CB catheter.

The current study has a few limitations. Given the underlying retrospective observational study design, selection bias could not be directly controlled for in our study. To alleviate selection bias, we used propensity matching, which allowed us to balance the two groups on study covariates. However, unmeasured confounders could still have influenced study results. Though not a complete substitute of randomised clinical trials (RCTs), it has been reported that treatment effects from observational studies using propensity scoring are similar to RCTs. ${ }^{23}$ However, it has been noted that propensity score matching can also increase imbalance and introduce bias. ${ }^{24}$ In addition, the loss of sample due to calliper matching, ${ }^{25}$ as also occurred in this study, could further add bias and limit the generalisability of study results. In our study, we observed a $~ 50 \%$ loss (1720 STSF patients after applying study criteria and 848 after propensity matching) of sample for the STSF group and $\sim 35 \%$ for the AFA-CB cohort (1295 AFA-CB patients after applying study criteria and 848 after propensity matching) for cost outcome assessment. When comparing the study characteristics of overall patients across (combined) the two cohorts who were not included in matched sample (1319 patients) with study characteristics of patients included in the matched cohorts (1696 patients), significant differences were observed. Patients who were not included in matching were observed to be sicker compared with those included in the matched sample, with significantly higher proportion of the unmatched patients having a CCI score 2 and above $(26.61 \%$ vs $22.17 \%, \mathrm{p}=0.0047)$, sleep apnoea $(28.81 \%$ vs $23.29 \%$; $\mathrm{p}=0.0006)$, diabetes $(22.52 \%$ vs $19.28 \%, \mathrm{p}=0.0296)$, heart failure $(24.87 \%$ vs $19.22 \%$, $\mathrm{p}=0.0002)$, atrial flutter $(48.52 \%$ vs $27.89 \%, \mathrm{p}<0.0001)$, other arrhythmia $(19.33 \%$ vs $16.51 \%, \mathrm{p}=0.0441)$, valvular disease $(24.94 \%$ vs $12.56 \%, \mathrm{p}<0.0001)$ and cardiomyopathy $(13.80 \%$ vs $11.20 \%, \mathrm{p}=0.0316)$. Considering these differences, and the potential for bias that could be introduced because of exclusion of patients from matching, we performed multivariable regression (GEE) analyses as secondary sensitivity analysis with the full sample identified through study inclusion/exclusion criteria (STSF=1720; $\mathrm{CB}=1295$ ) for the assessment of hospital cost outcome. All study covariates were adjusted for in the GEE (log link and gamma distribution) model to examine total cost and supply cost. The results from multivariable GEE analyses showed total cost to be $\sim 13 \%$ lower (exponentiated ratio (ER) $0.87,95 \%$ CI 0.83 to 0.92 ) and supply cost to be $\sim 24 \%$ lower (ER $0.76,95 \%$ CI 0.68 to 0.85 ) for STSF compared with the AFA-CB catheter. The results from the multivariable GEE analyses were consistent with the propensity score matching results where STSF use was associated with significantly lower total cost and supply cost compared with the AFA-CB catheter use. As done in our study, using a retrospective database that is nationally representative along with propensity matching technique likely alleviates some of the main limitations associated with observational studies. Some of the core clinical parameters like AF severity or PVs isolated, concomitant antiarrhythmic drug or oral anticoagulant drug use, and procedural elements including fluoroscopy time and ablation time were not available in the database and could not be compared between the two technologies. However, it should be noted that procedural elements like fluoroscopy time and ablation time, though important, are unlikely to have an effect on the long-term outcomes that were considered in this study. Further, in PHD, readmission can only be assessed if the patient was to come back to the same hospital. Readmission cannot be assessed if a patient goes to a different hospital irrespective of whether or not the different hospital provides data to PHD or not. As a result, the rate of readmission in our study could be lower than the true rate of readmission. In addition, to be able to assess readmission, it is imperative that the hospital continues to provide data to PHD. To minimise this limitation, we restricted our readmission analysis only to those patients who had index ablation in a hospital that continued to provide data to PHD for the subsequent 6-month follow-up period. We identified device use based on a text search strategy, as unique device identifiers (UDIs) were missing. As hospital billing systems increasingly adopt UDIs, which have been implemented by the Food and Drug Administration to adequately identify devices in the USA, it is likely to enable researchers to have structured and objective criteria for device identification. Until that time, while not without flaws, the use of text search strategy remains a feasible alternative to device identification. Given that the STSF catheter was released in the latter half of 2016, and with data availability limited to first half of 2018, we could not study long-term outcomes among patients in the study. Further research is needed to better understand if the short-term outcome (postblanking 4-6 months) differential between STSF and CB catheter translates to long-term outcomes (postblanking 4-12 months) as well. Lastly, any potential errors that occurred during billing or during claims coding could have influenced study results.

\section{CONCLUSION}

Study results showed significantly lower total index ablation and supply costs, and a significantly lower likelihood of CV-related readmission in the postblanking period 
(4-6 months) among patients with AF who had an ablation with the STSF catheter compared with the CB catheter. With the combination of the advanced porous tip and CF technology, the STSF catheter offers considerable economic savings to providers and significant health benefit to patients compared with the AFA-CB catheter. Further research is needed to determine if the results observed in this study hold consistent over a longer postblanking follow-up period.

\section{Author affiliations}

${ }^{1}$ Department of Internal Medicine, Center for Electrophysiology, Rhythm Disorders and Electro-Mechanical Interventions, University of Cincinnati, Cincinnati, Ohio, USA ${ }^{2}$ Franchise Health Economics and Market Access, Johnson and Johnson Medical Devices, Irvine, California, USA

${ }^{3}$ Medical Device Epidemiology, Johnson and Johnson Medical Devices, New Brunswick, New Jersey, USA

Acknowledgements Medical writing services were provided by Susan BartkoWinters, PhD, from SBW Medical Writing Inc, which received funding from Johnson and Johnson.

Contributors AC, LG, SM, IK and RK were involved in the conception and design of the study. IK and RK led the supervision of data collection and led data analysis and AC, LG, SM, IK and RK were involved in data interpretation. RK led the development of the first draft of the manuscript, which was then refined based on input and comments from AC, LG, SM and IK. AC, LG, SM, IK and RK approved the final version of the manuscript.

Funding This study was sponsored by Johnson and Johnson.

Competing interests $\mathrm{AC}$ has been a paid speaker for Biosense Webster (BWI) and BIOTRONIK. RK, SM, IK and LG are Johnson and Johnson employees.

Patient consent for publication Not required.

Ethics approval Retrospective analysis of data from the Premier Healthcare Database, as conducted in this study, are considered exempt from Institutional Review Board (IRB) oversight as dictated by Title 45 Code of Federal Regulations, Part 46 of the USA, specifically 45 CFR 46.101(b)(4) (http://www.hhs.gov/ohrp/ humansubjects/guidance/45cfr46.html).

Provenance and peer review Not commissioned; externally peer reviewed.

Data availability statement Data may be obtained from a third party and are not publicly available. The data used for the purposes of this study are not publicly available, however, researchers could avail the data through agreement with thirdparty vendor.

Open access This is an open access article distributed in accordance with the Creative Commons Attribution Non Commercial (CC BY-NC 4.0) license, which permits others to distribute, remix, adapt, build upon this work non-commercially, and license their derivative works on different terms, provided the original work is properly cited, appropriate credit is given, any changes made indicated, and the use is non-commercial. See: http://creativecommons.org/licenses/by-nc/4.0/.

ORCID iD

Rahul Khanna http://orcid.org/0000-0002-2478-1724

\section{REFERENCES}

1 Wolf PA, Abbott RD, Kannel WB. Atrial fibrillation as an independent risk factor for stroke: the Framingham study. Stroke 1991;22:983-8.

2 Wilber DJ, Pappone C, Neuzil P, et al. Comparison of antiarrhythmic drug therapy and radiofrequency catheter ablation in patients with paroxysmal atrial fibrillation: a randomized controlled trial. JAMA 2010;303:333-40.

3 Packer DL, Mark DB, Robb RA, et al. Effect of catheter ablation vs antiarrhythmic drug therapy on mortality, stroke, bleeding, and cardiac arrest among patients with atrial fibrillation: the CABANA randomized clinical trial. JAMA 2019;321:1261-74.
4 Calkins H, Hindricks G, Cappato R, Badhwar V, Brugada J, Camm J, et al. $2017 \mathrm{HRS} / \mathrm{EHRA} / \mathrm{ECAS/APHRS/SOLAECE} \mathrm{expert} \mathrm{consensus}$ statement on catheter and surgical ablation of atrial fibrillation: executive summary. J Interv Card Electrophysiol 2017:50:1-55.

5 Maurer T, Rottner L, Makimoto H, et al. The best of two worlds? pulmonary vein isolation using a novel radiofrequency ablation catheter incorporating contact force sensing technology and 56-hole porous tip irrigation. Clin Res Cardiol 2018;107:1003-12.

6 Fürnkranz A, Bordignon S, Dugo D, et al. Improved 1-year clinical success rate of pulmonary vein isolation with the second-generation cryoballoon in patients with paroxysmal atrial fibrillation. $J$ Cardiovasc Electrophysiol 2014;25:840-4.

7 Martins RP, Hamon D, Césari O, et al. Safety and efficacy of a second-generation cryoballoon in the ablation of paroxysmal atrial fibrillation. Heart Rhythm 2014;11:386-93.

8 Jourda F, Providencia R, Marijon E, et al. Contact-force guided radiofrequency vs. second-generation balloon cryotherapy for pulmonary vein isolation in patients with paroxysmal atrial fibrillation-a prospective evaluation. Europace 2015;17:225-31.

9 Pollak SJ, Goldstein L, Daskiran M, et al. Economic impact of atrial fibrillation ablation with radiofrequency contact force catheter versus cryoballoon catheter. J Comp Eff Res 2019;8:251-64.

10 Kuck K-H, Brugada J, Fürnkranz A, et al. Cryoballoon or radiofrequency ablation for paroxysmal atrial fibrillation. $N$ Engl $J$ Med 2016;374:2235-45.

11 Kuck K-H, Brugada J, Schlüter M, et al. The fire and ice trial: what we know, what we can still learn, and what we need to address in the future. J Am Heart Assoc 2018;7:e010777.

12 Mandell J, Amico F, Parekh S, et al. Early experience with the cryoablation balloon procedure for the treatment of atrial fibrillation by an experienced radiofrequency catheter ablation center. J Invasive Cardiol 2013;25:288-92.

13 Aryana A, Singh SM, Kowalski M, et al. Acute and long-term outcomes of catheter ablation of atrial fibrillation using the secondgeneration Cryoballoon versus Open-Irrigated radiofrequency: a multicenter experience. J Cardiovasc Electrophysiol 2015;26:832-9.

14 Morillo CA, Banerjee A, Perel P, et al. Atrial fibrillation: the current epidemic. J Geriatr Cardiol 2017;14:195-203.

15 Deyo RA, Cherkin DC, Ciol MA. Adapting a clinical comorbidity index for use with ICD-9-CM administrative databases. J Clin Epidemiol 1992;45:613-9.

16 Quan $\mathrm{H}$, Li B, Couris CM, et al. Updating and validating the Charlson comorbidity index and score for risk adjustment in hospital discharge Abstracts using data from 6 countries. Am J Epidemiol 2011;173:676-82.

17 Austin PC, Stuart EA. Moving towards best practice when using inverse probability of treatment weighting (IPTW) using the propensity score to estimate causal treatment effects in observational studies. Stat Med 2015;34:3661-79.

18 Cappato R, Calkins H, Chen S-A, et al. Worldwide survey on the methods, efficacy, and safety of catheter ablation for human atrial fibrillation. Circulation 2005;111:1100-5.

19 Ciconte G, Velagić V, Mugnai G, et al. Electrophysiological findings following pulmonary vein isolation using radiofrequency catheter guided by contact-force and second-generation cryoballoon: lessons from repeat ablation procedures. Europace 2016;18:71-7.

20 Gunawardene MA, Hoffmann BA, Schaeffer B, et al. Influence of energy source on early atrial fibrillation recurrences: a comparison of cryoballoon vs. radiofrequency current energy ablation with the endpoint of unexcitability in pulmonary vein isolation. Europace 2018;20:43-9.

21 Kardos A, Kis Z, Som Z, et al. Two-year follow-up after contact force sensing radiofrequency catheter and second-generation Cryoballoon ablation for paroxysmal atrial fibrillation: a comparative single centre study. Biomed Res Int 2016;2016:1-6.

22 Zhou X, Lv W, Zhang W, et al. Comparative efficacy and safety of contact force-sensing catheter and second-generation cryoballoon ablation for atrial fibrillation: a meta-analysis. Braz J Med Biol Res 2017;50:e6409.

23 Collins GS, Le Manach Y. Comparing treatment effects between propensity scores and randomized controlled trials: improving conduct and reporting. Eur Heart J 2012;33:1867-9.

24 King G, Nielsen R. Why propensity scores should not be used for matching. Polit. Anal. 2019;27:435-54.

25 Austin PC. Double propensity-score adjustment: a solution to design bias or bias due to incomplete matching. Stat Methods Med Res 2017;26:201-22. 